\title{
Octupole Magnet For Soft X Ray Magnetic Dichroism Experiments: Design and Performance
}

\author{
Elke Arenholz ${ }^{*}$ and Soren O. Prestemon ${ }^{\dagger}$ \\ *Advanced Light Source, Lawrence Berkeley National Laboratory, Berkeley, CA \\ ${ }^{\dagger}$ Engineering Division, Lawrence Berkeley National Laboratory, Berkeley, CA
}

\begin{abstract}
An octupole magnet endstation for soft x ray magnetic dichroism measurements has been developed at the Advanced Light Source. The system consists of an eight pole electromagnet that surrounds a small vacuum chamber. The magnet provides fields up to $0.9 \mathrm{~T}$ that can be applied in any direction relative to the incoming $\mathrm{x}$ ray beam. High precision magnetic circular and linear dichroism spectra can be obtained reversing the magnetic field for each photon energy in an energy scan. Moreover, the field dependence of all components of the magnetization vector can be studied in detail by choosing various angles of $\mathrm{x}$ ray incidence while keeping the relative orientation of magnetic field and sample fixed.
\end{abstract}

\section{MOTIVATION}

Soft $\mathrm{x}$ ray magnetic dichroism (SX-MD) has become a powerful and important tool for the study of magnetic materials [1]. It is element-specific, in some cases even site-specific, provides sub-monolayer sensitivity and is therefore ideally suited for the study of multilayered, multi-element, and heteromagnetic nanostructures relevant for magnetic data storage applications today.

Soft x-ray magnetic circular dichroism (SX-MCD) probes the projection of the magnetization vector $\mathbf{M}$ along the direction of the photon momentum $\mathbf{k}$ of the incoming $\mathrm{x}$ ray beam. SX-MCD spectra can be determined with $\mathrm{x}$ rays of fixed circular polarization by reversing the sample magnetization from parallel to antiparallel relative to $\mathbf{k}$ with an external magnetic field. Since SX-MCD is proportional to $\mathbf{k} \cdot \mathbf{M}$ all components of the magnetization vector and their change with applied field can be determined precisely by varying the $\mathrm{x}$ ray incidence angle while keeping the relative orientation of magnetic field and sample constant [2]. However, this requires a magnet that can provide variable magnetic fields in three dimensions.

Soft x-ray magnetic linear dichroism (SX-MLD) is proportional to the orientation of the magnetic moment $\mathbf{M}$ relative to the polarization of the incident $\mathrm{x}$ ray beam $\varepsilon$, i.e. $\left\langle(\mathrm{M} \cdot \varepsilon)^{2}\right\rangle$. Since SX-MLD in contrast to SX-MCD does not require a net magnetization it can be used to study magnetic phenomena in antiferromagnetic systems. In ferromagnetic systems, SX-MLD can be measured by switching the magnetization between parallel and perpendicular relative to the polarization direction with an external magnetic field. Antel et al. [3] have shown that inplane magnetic anisotropies can be characterized by monitoring the SX-MLD effect while rotating the magnetic field in the sample plane. Again the control of the external magnetic field in three dimensions is necessary.

To take full advantage of the strengths of SX-MD measurements for the detailed characterization of complex magnetic materials we developed an eight pole electromagnet end station that provides magnetic fields up to $0.9 \mathrm{~T}$ in three dimensions.

\section{DESIGN CONSIDERATIONS}

Superconducting octupole magnets [4] and permanent magnet devices [5] that provide variable magnetic fields in three dimensions have been proposed and built in the past. To reverse the magnetic field in these instruments typically takes tens of seconds to tens of minutes making it impractical to flip the field for each photon energy in an 


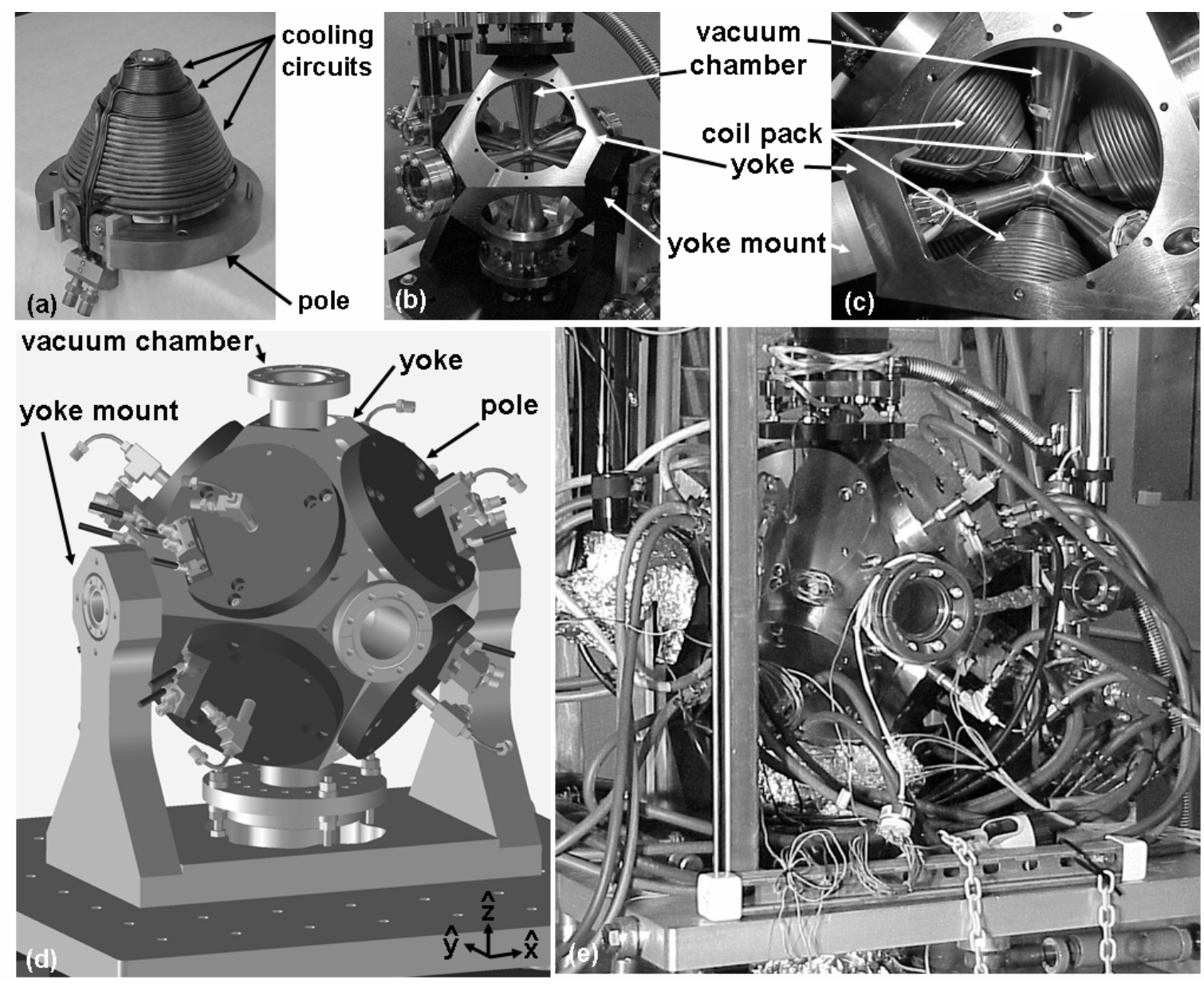

FIGURE 1. Photographs and design sketch of octupole magnet. (a) Coil pack with three cooling circuits and pole. (b) Magnet yoke with Ti vacuum chamber supported by yoke mount with all eight poles removed. (c) View inside the magnet yoke with only one pole removed. (d) Design sketch of octupole magnet endstation. (e) Photograph of completely assembled endstation.

energy scan. Since the precision with which small SX-MD effects can be determined is increased significantly by reversing the field point by point [6] we chose to design and build an electromagnet allowing field reversal in $<0.5 \mathrm{~s}$.

The design aimed at maximum magnetic fields of $\mathrm{B} \geq 0.8 \mathrm{~T}$ for the typical duration of a $\mathrm{x}$ ray absorption scan. The field uniformity was required to be better than $1 \%$ within $2.5 \mathrm{~mm}$ of the magnet center to allow sufficient homogeneity of the magnetic field over the $\mathrm{x}$ ray spot even for extreme grazing angles of $\mathrm{x}$ ray incidence onto the sample. Although three orthogonal electromagnet dipole pairs would be sufficient to provide the desired fields we chose an eight pole configuration. It is easier to achieve the required field homogeneity with the octupole configuration and typically requires fewer A-turns per pole for the same field than a sextupole.

The magnet coils are not directly wound onto the poles but on aluminum mandrels which are then in turn bolted onto the magnet poles. This allows the use of different pole materials optimizing the setup for maximum field (steel poles) or highest field precision around zero field (non-magnetic aluminum poles). The current carrying $\mathrm{Cu}$ wire has a square cross section $\left(2 \times 2 \mathrm{~mm}^{2}\right)$ and is wound with 180 turns per coil. For maximum magnetic field $(\mathrm{B} \geq 0.8 \mathrm{~T})$ more than $3 \mathrm{~kW}\left(\mathrm{I}=100 \mathrm{~A}, \mathrm{R}_{\text {coil }} \approx 0.35 \Omega\right.$ ) are dissipated in each coil. To avoid overheating the coils are covered with three parallel water cooling circuits and a squirt tube inserted into each pole. A theoretical thermal analysis of the coil pack/pole assembly and testing of a prototype prior to fabrication of the entire system ensured that the cooling is sufficient to reach fields of $0.9 \mathrm{~T}$.

Magnetic flux closure is achieved through a steel box yoke that holds all eight poles. The shared yoke does not appear to significantly affect the performance and simplifies the design. The dimensions of the magnet yoke and the magnet poles (taper, diameter, length) were carefully optimized using the software package TOSCA (by Vector Fields Inc., Oxford, UK) to meet the specifications for field strength and homogeneity compatible with cooling 

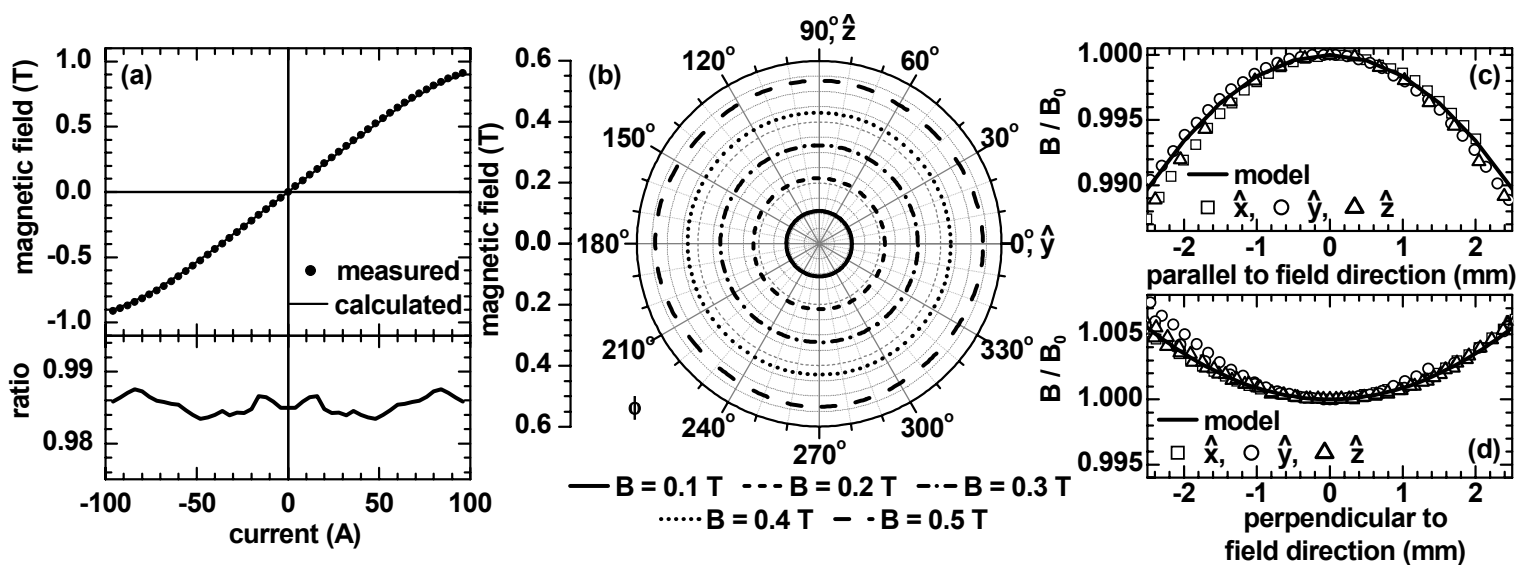

FIGURE 2. Magnetic performance of the octupole magnet. (a) Calculated and measured current-field relationship for magnetic fields applied along the principle axes $\mathbf{x}, \mathbf{y}, \mathbf{z}$ of the magnet. (b) Field vector for magnetic fields up to $0.5 \mathrm{~T}$ of rotational loops in the yz plane. (c, d) Spacial variation of magnetic fields along (c) and perpendicular (d) to the field direction for fields applied in the principle directions $\mathbf{x}, \mathbf{y}$, and $\mathbf{z}$.

requirements and space constraints. Design optimization studies showed that fields in the magnet center can be significantly increased by covering the pole tip region with the coil. The pole taper angle was chosen so as to ensure that the pole-tip saturates before the rest of the pole as current is increased. Also, increasing the length of the coil/pole assemblies while reducing the diameter but keeping the same A-turns produces nearly the same center field and allows for better cooling performance.

The magnet was designed to surround a small non-magnetic Ti-alloy vacuum chamber. Placing the magnet outside vacuum simplifies design, fabrication, and use of the endstation substantially. Since the magnetic field decreases rapidly with increasing pole distance the design aimed at minimizing the pole gap while providing enough space for the vacuum chamber. The center sphere of the chamber has a diameter of 2 inches and can be reached through six access ports that have a minimum diameter of 0.8 inch (ID). A sample holder that allows polar and azimuthal rotation is inserted from the top. Sample temperatures between $17 \mathrm{~K}$ and $450 \mathrm{~K}$ have been achieved.

The coils, poles, the yoke and the chamber are shown in Figure 1.

\section{MAGNETIC PERFORMANCE}

The magnetic performance of the octupole magnet was characterized by inserting a three axis Hall transductor (3R100-3-2 by Sentron, Switzerland) from the top into the magnet in position of the sample. Figure 2(a) shows the measured and calculated current-field relationship for currents up to almost $100 \mathrm{~A}$ and field vectors aligned along the principle axes $\mathbf{x}, \mathbf{y}, \mathbf{z}$ as defined in Figure 1(d). The agreement is within 1.5\% over the entire current range. Part of the systematic deviation could be due to the $1 \%$ uncertainty in the absolute current calibration.

$\mathrm{Up}$ to $50 \mathrm{~A}$ the increase of the magnetic field with current is linear within $0.5 \%$. Using this linear current-field relationship we calculated the currents required to rotate the magnetic field vector within the yz-plane and measured the actual field vectors. The results are plotted in Figure 2(b). The magnetic field strength is within $1.5 \%$ and its orientation within $1^{\circ}$ of the desired values for fields up to $0.5 \mathrm{~T}$. For larger fields the deviations increase due to the increasing importance of non-linear terms in the current-field relation. It is possible to obtain magnetic field vectors more precisely by including the non-linear terms.

For field vectors oriented along the principle axes of the magnet we determined the special variation along and perpendicular to the field direction. The results for $\mathrm{B}=0.4 \mathrm{~T}$ are shown in Figure 2(c) and (d), respectively. The uniformity of the field is indeed better than $1 \%$ close to the magnet center. The model calculations predict the shape of the field profile within $0.5 \%$ and its absolute value within $1.5 \%$.

\section{FIRST SPECTROSCOPY RESULTS}

The octupole magnet endstation has been installed at beamline 4.0.2 [7] at the Advanced Light Source. The beamline is equipped with an elliptically polarizing undulator and provides left and right circular as well as linear 

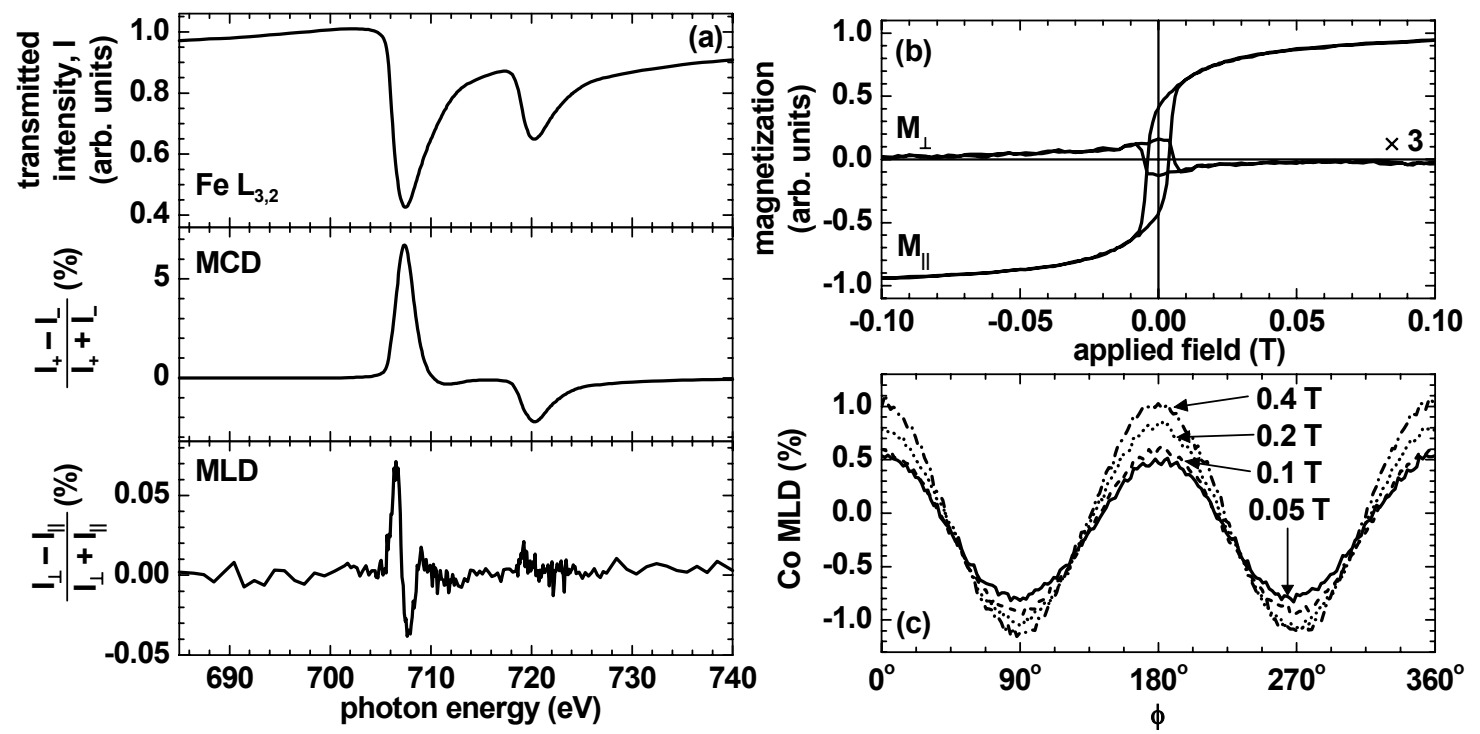

FIGURE 3. (a) SX-MD at the Fe $\mathrm{L}_{3,2}$ edges of a $40 \mathrm{~nm}$ Terfenol film. Top: Transmission spectrum. Center: Transmission SXMCD spectrum. Bottom: Transmission SX-MLD spectrum. (b) In-plane magnetization reversal in a $40 \mathrm{~nm}$ Terfenol film as determined by Dy $\mathrm{M}_{5} \mathrm{SX}-\mathrm{MCD}$. (c) $\mathrm{Co}_{3} \mathrm{SX}-\mathrm{MLD}$ rotational loops obtained from a Co/Terfenol multilayer.

horizontal to linear vertical polarization from $80 \mathrm{eV}$ to $2000 \mathrm{eV}$. As a first demonstration of the capabilities of the endstation we determined the Fe $\mathrm{L}_{3,2}$ SX-MCD and SX-MLD spectra in a transmission experiment on a $40 \mathrm{~nm}$ Terfenol film in normal incidence geometry. The transmission spectrum is shown in the top panel of Figure 3(a). The SX-MCD spectrum displayed in the center panel was measured with fixed circular polarization by switching the field orientation between parallel and antiparallel to the photon momentum for each photon energy. The SX-MLD spectrum was determined with fixed linear polarization by switching the magnetic field between perpendicular and parallel relative to the polarization direction at each photon energy. Although the change in transmitted intensity is only $0.05 \%$ it is possible to characterize the effect precisely with our system.

Element-specific magnetic hystersis curves were determined by monitoring the peak intensity of the Dy $\mathrm{M}_{5}$ absorption line as function of applied field. Following the procedure outline by Chakarian et al. [2] the magnetization reversal for fields within the sample plane was determined and is shown in Figure 3(b).

Figure 3(c) displays the change in the $\mathrm{Co}_{3} \mathrm{SX}-\mathrm{MLD}$ signal of a multilayer consisting of three repeats of $20 \mathrm{~nm}$ Co and $10 \mathrm{~nm}$ Terfenol measured in normal incidence geometry when the magnetic field is rotated within the sample plane. Since the sample does not show any inplane anisotropy the magnetization follows the applied field and the amplitude of the SX-MLD signal follows $\cos (2 \phi)$, where $\phi$ is the angle between magnetization and polarization vector.

\section{ACKNOWLEDGMENTS}

The Terfenol samples were provided by P. Fischer, Max-Plank-Institut, Stuttgart, Germany. The Advanced Light Source is supported by the Director, Office of Basic Energy Sciences, of the U.S. Department of Energy.

\section{REFERENCES}

1. J. Stöhr, Magn. Magn. Mater. 75, 253-272 (1995).

2. V. Chakarian et al., Appl. Phys. Lett. 66, 3368-3370 (1995).

3. J.W. Antel et al., Appl. Phys. Lett. 79, 997-999 (2001).

4. C.S. Hwang et al., J. Magn. Magn. Mater. 239, 586-590 (2002).

5. C.S. Hwang et al., J. Magn. Magn. Mater. 209, 169-172 (2000).

6. E. Dudzik et al., SRN 13,18-22 (2000).

7. A.T. Young et al., Nucl. Instr. and Meth.A 467-468, 549-552 (2001). 